\title{
Síndrome POEMS: mujer de 60 años con adenopatías múltiples, polineuropatía y enfermedad de Castleman
}

\section{POEMS syndrome: 60-year-old woman with multiple adenopathies, polyneuropathy and Castleman's disease.}

\author{
Eloy Peña,' Sonia Indacochea,' Daniel Angulo,' \\ Richard Rubio' y Juan Diego Podestá ${ }^{2}$
}

Peña E, Indacochea S, Angulo D, Rubio R, Podestá JD. Síndrome POEMS: mujer de 60 años con adenopatías múltiples, polineuropatía y enfermedad de Castleman. Rev Soc Peru Med Interna. 2019;32(3): 107-III. https://doi.org//0.36393/spmi.v32i3.477

\begin{abstract}
RESUMEN
Se reporta el caso de una paciente mujer de 60 años, con seis meses de enfermedad, caracterizado por adenopatías axilares, debilidad generalizada ascendente e hiperpigmentación de piel. Los estudios de imágenes mostraron adenopatías cervicales, axilares y mediastinales, hepatoesplenomegalia, lesiones osteoblásticas en fémur y arco costal. La electromiografía mostró polineuropatía periférica sensitivo-motora de tipo axonal. La inmunofijación en suero reveló componente monoclonal de clase lgG tipo lambda y la biopsia de ganglio axilar presentó hallazgos consistentes con enfermedad de Castleman, por lo que el diagnóstico final fue síndrome POEMS.

Palabras Clave. síndrome de POEMS, enfermedad de Castleman, gammapatía monoclonal, polineuropatía.
\end{abstract}

\section{ABSTRACT}

We report the case of a 60-year old female patient with 6-month disease time characterized by axillary adenopathy, generalized ascending weakness and hyperpigmentation of the skin. Imaging studies showed cervical, axillary, mediastinal adenopathy, hepatosplenomegaly, osteoblastic lesions in femur and costal arch. Electromyography showed axonal-type motor peripheral sensory polyneuropathy. Serum immunofixation revealed monoclonal component of Lambda type IgG class and axillary lymph node biopsy showed findings consistent with Castleman disease; so, the final diagnosis was POEMS syndrome.

KEYWORDS: POEMS syndrome, Castleman disease, monoclonal gammopathy, polyneuropathy.

\section{INTRODUCCIÓN}

Las gammapatías monoclonales comprenden varias enfermedades como amiloidosis de cadenas ligeras (AL), gammapatía monoclonal de significado indeterminado (MGUS), mieloma múltiple (MM), macroglobulinemia de Waldestrom y síndrome POEMS. ${ }^{1}$ Tienen una

I. Médico internista. Servicio de Medicina Interna, Hospital Nacional Essalud Edgardo Rebagliati Martins, Lima.

2. Médico residente de Medicina interna. Servicio de Medicina Interna, Hospital Nacional Essalud Edgardo Rebagliati Martins, Lima. prevalencia de $3 \%$ a $4 \%$ en mayores de 50 años y de $5 \%$ en mayores de 70 años. ${ }^{2}$

El POEMS (polineuropatía, organomegalia, endocrinopatía, cambios en la piel) es un síndrome paraneoplásico raro, secundario al trastorno de las células plasmáticas. ${ }^{3}$ Afecta a 0,3 por 100000 habitantes y su incidencia máxima se encuentra en la quinta y sexta décadas de la vida. $^{4}$

La presencia de polineuropatía más cualquiera de los siguientes hallazgos: proteína monoclonal, trombocitosis, anasarca, papiledema y polineuropatía desmielinizante inflamatoria crónica que no responde al tratamiento debe hacer pensar en POEMS. Una buena historia clínica y un minucioso examen físico, seguidos de exámenes auxiliares adecuados como evaluación radiográfica de huesos, medición sérica de factor de crecimiento de endotelio vascular (VEGF) y biopsia de médula ósea, pueden diferenciar el POEMS de otras gammapatías monoclonales. ${ }^{5-8}$

Existe una variante de la enfermedad de Castleman (EC) con POEMS que no presenta proliferación de células plasmáticas clonales, pero sí muchas de las 
otras características paraneoplásicas. ${ }^{9}$ La EC puede estar asociada o no al POEMS. La EC se clasifica en unicéntrica y multicéntrica. La multicéntrica puede cursar con neuropatía periférica, que, a diferencia de la neuropatía en el POEMS, es sensorial, distal y leve.

\section{PRESENTACIÓN DEL CASO}

Paciente mujer de 60 años, natural de Ica y procedente de Lima, ocupación ama de casa, raza mestiza, con antecedente de sinusitis crónica hace 15 años. Con seis meses de evolución, de curso insidioso y progresivo, inició con aparición de tumoración axilar bilateral de aproximadamente $2 \mathrm{~cm}$, no dolorosas y poco blandas. Cinco meses antes del ingreso, se agregó parestesias y aumento de volumen en los miembros inferiores, que, posteriormente, progresan a debilidad muscular de esas extremidades, que, después, asciende a miembros superiores. Tres meses antes del ingreso, notó coloración oscura en piel de manera generalizada, la cual aumentó en intensidad en el tiempo. Un mes antes del ingreso, la debilidad generalizada era marcada y cursaba con dolor urente en las extremidades, con dificultad para la bipedestación. Dos semanas antes del ingreso, acudió a un hospital, donde le realizan biopsia de la tumoración axilar y la informan como cambios reactivos y folículos con morfología similar a EC tipo hialino-vascular, por lo que es referida a nuestro nosocomio, para continuar el diagnóstico y determinar el tratamiento.

\section{Examen físico}

$\mathrm{Al}$ ingreso, la paciente estaba lúcida, con funciones vitales estables. Con hiperpigmentación generalizada de la piel, sin compromiso de mucosas, edema bilateral moderado de miembros inferiores hasta las rodillas (Figura 1). Dolor moderado a la palpación en las extremidades, sin compromiso articular. Ganglios axilares bilaterales de cerca de $2 \mathrm{~cm}$, poco blandos, no dolorosos y móviles. Se logra palpar borde hepático a $2 \mathrm{~cm}$ debajo del reborde costal derecho, bazo no palpable. Al examen neurológico, fuerza muscular disminuida (3/5), de predominio distal simétrico, hiporreflexia generalizada y sensibilidad disminuida de manera generalizada a nivel periférico. Resto del examen clínico sin alteraciones.

\section{Exámenes auxiliares}

Hemoglobina $17 \mathrm{~g} / \mathrm{dL}$, leucocitos $9110 / \mathrm{mm}^{3}$ (neutrófilos $58,2 \%$, linfocitos $30,8 \%$ ) y plaquetas en $329000 / \mathrm{mm}^{3}$; proteínas totales $5,8 \mathrm{~g} / \mathrm{dL}$, albúmina $2,9 \mathrm{~g} / \mathrm{dL}$; fosfatasa alcalina $157 \mathrm{UI} / \mathrm{L}$, bilirrubinas normales.
Examen de orina, electrólitos y perfiles renal, lipídico y de coagulación en rangos normales.

TSH 6,63 (VN 0,4-4 $\mu \mathrm{UI} / \mathrm{mL}$ ), T4 libre 1,12 (VN 0,81,9 ng/dL), T3 libre 1,37 (VN 1,8-4,2 pg/mL), PTH $1,8 \mathrm{pg} / \mathrm{mL}$ (VN 11-67 pg/mL), prolactina 22,9 (VN $<25 \mathrm{ng} / \mathrm{mL}$ ), FSH $<0,1$ (VN 25-134 mUI/mL), LH $<0,1$ (VN 7-58 mUI/mL), HbA1C 6,1 \%, insulina basal 10,8 (VN 9,3-29,1 uUI/mL), péptido $\mathrm{C}$ basal 3,92 (VN 0,9-7,1 ng/mL), ACTH 20,12 (VN hasta $46 \mathrm{pg} / \mathrm{mL}$ ) y cortisol 10,1 (VN 5-25 $\mu \mathrm{g} / \mathrm{dL})$.

Virus Epstein-Barr, TORCH, HTLV I-II, VIH, virus herpes humano tipo 8 (VHH-8), marcadores tumorales, DHL, $\beta 2$ microglobulina y baciloscopia para tuberculosis negativos.

El estudio tomográfico (TAC) evidenció ganglios axilares bilaterales a predominio derecho, siendo el mayor de $28 \mathrm{~mm}$, además de hepatoesplenomegalia, quistes hepáticos simples y un nódulo pulmonar de $6 \mathrm{~mm}$ en segmento posterior del lóbulo posterior derecho no calcificado.

Electromiografía: signos compatibles con polineuropatía avanzada sensitivo-motora de tipo axonal, activa y con escasos signos de reinervación.

Electroforesis de proteínas en suero: presencia de banda a nivel de tercio distal de fracción gamma, con componente monoclonal de clase IgG tipo lambda.

TAC de cerebro, tórax, abdomen y pelvis con contraste: nódulo de $6 \mathrm{~mm}$ en el segmento posterior del lóbulo posterior derecho no calcificado; ganglios axilares, el derecho de $28 \mathrm{~mm}$; hígado con numerosas formaciones quísticas simples, ninguna lesión sólida (Figura 2).

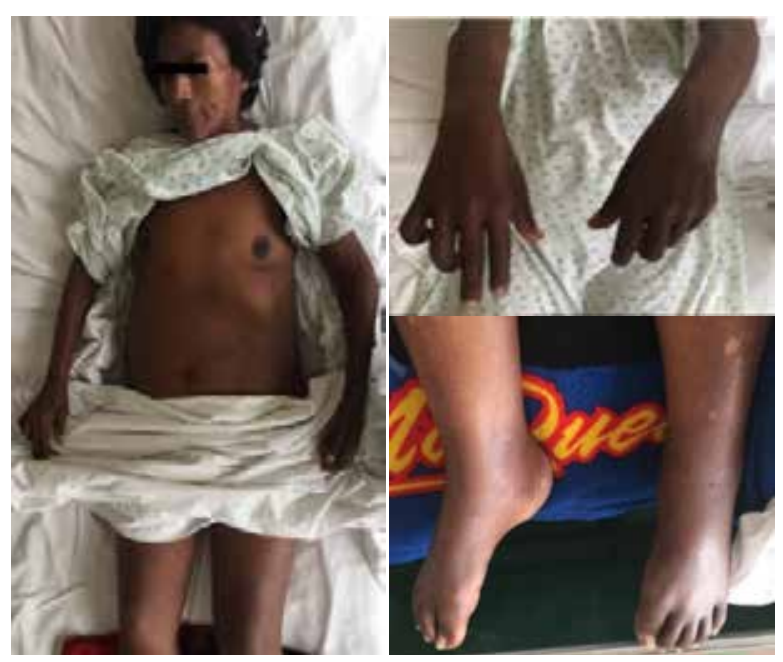

Figura I. Hiperpigmentación generalizada de la piel y edema blando de los miembros inferiores. 
Gammagrafía ósea: hipercaptación patológica en tórax y fémures, sugerentes de lesiones osteoblásticas; corroboradas con PET scan, con hipermetabolismo focal de localización intramedular en los dos tercios proximales de fémur izquierdo y lesión blástica sin metabolismo en el 12. ${ }^{\circ}$ arco costal posterior izquierdo. (Figura 3).

Estudio de médula ósea: celularidad de $40 \%$ con plasmocitosis atípica (células plasmáticas en $10 \%$ ) e hiperplasia megacariocítica.

Biopsia de ganglio axilar: folículos linfoides hipertróficos e hipotróficos, hiperplasia concéntrica de la zona del manto y presencia de células plasmáticas en los cortes germinales y el área interfolicular (Figura 4). La inmunohistoquímica mostró presencia de células $\mathrm{B}$ y $\mathrm{T}$ en sus respectivos compartimientos, sin evidencia de proliferación neoplásica, por lo que se concluyó en hallazgos consistentes con EC, variante plasmocelular.

\section{DISCUSIÓN}

En 1954, Castleman describió a un hombre de 40 años, con historia de fiebre de varios años, tos no productiva y dolor torácico a quien se le encontró una masa en el mediastino; en la escisión quirúrgica, la patología mostró linfoproliferación. ${ }^{10}$ Este caso seguidos de otros 12 pacientes informados por Castleman es lo que ahora se conoce como EC unicéntrica (ECU). ${ }^{11}$

La EC en un trastorno linfoproliferativo raro, caracterizado por hiperplasia angiofolicular del nódulo linfático. Comprende dos categorías, la ECU -linfadenopatía solitaria- y la EC multicéntrica -múltiples linfadenopatías (ECM)-. La ECM puede estar asociada a virus herpes humano tipo 8 (VHH-8) o ser VHH-8 negativo. La ECM que es VHH-8 negativo se subdivide en la asociada a POEMS y la ECM idiopática (ECMi). Esta última puede estar asociado a trombocitopenia, anasarca, mielofibrosis, falla renal y organomegalia (TAFRO). ${ }^{12}$

La ECU puede presentarse a cualquier edad, la mediana de edad es de 30 a 34 años, con un ligero predominio femenino $(60 \%)$. La incidencia es de 15 a 19 pacientes por un millón de personas-año, y $95 \%$ de casos tienen histología de tipo hialino vascular. No hay asociación con infección por VIH o VHH-8 y no se han identificado factores de riesgo epidemiológicos. La ECM tiene una prevalencia total de 21 a 25 por millón de personas-año, La incidencia de ECMi es de 5 por un millón al año, pero con una variación regional importante. La ECM más VHH-8 es más común en pacientes infectados por VIH o cualquier otra causa de inmunosupresión. ${ }^{13}$

La ECU está limitada a una única cadena o área ganglionar. Por lo general es asintomática, por lo que el ganglio afectado se descubre incidentalmente. Los síntomas que podrían presentarse son por afectación local de la tumoración y, en los casos excepcionales en que hay síntomas sistémicos, probablemente estén relacionados al incremento de la interleucina 6 (IL6). El ganglio afectado tiene un tamaño promedio de $5,5 \mathrm{~cm}$; $29 \%$ se encuentran en el mediastino; $23 \%$, en el cuello; $21 \%$, en el retroperitoneo; $17 \%$, en otras áreas.

El diagnóstico se hace con anatomía patológica. La extirpación del ganglio casi siempre es curativa pero se han reportado recurrencias, y no se han reportado casos de transición a ECM. Existen teorías inflamatorias, virales y neoplásicas no corroboradas para explicar la patogénesis. La célula responsable y las vías de señalización específica no han sido identificadas. ${ }^{14}$

La ECM afecta múltiples localizaciones, cursa con hepatoesplenomegalia, fiebre y sudoración nocturna,

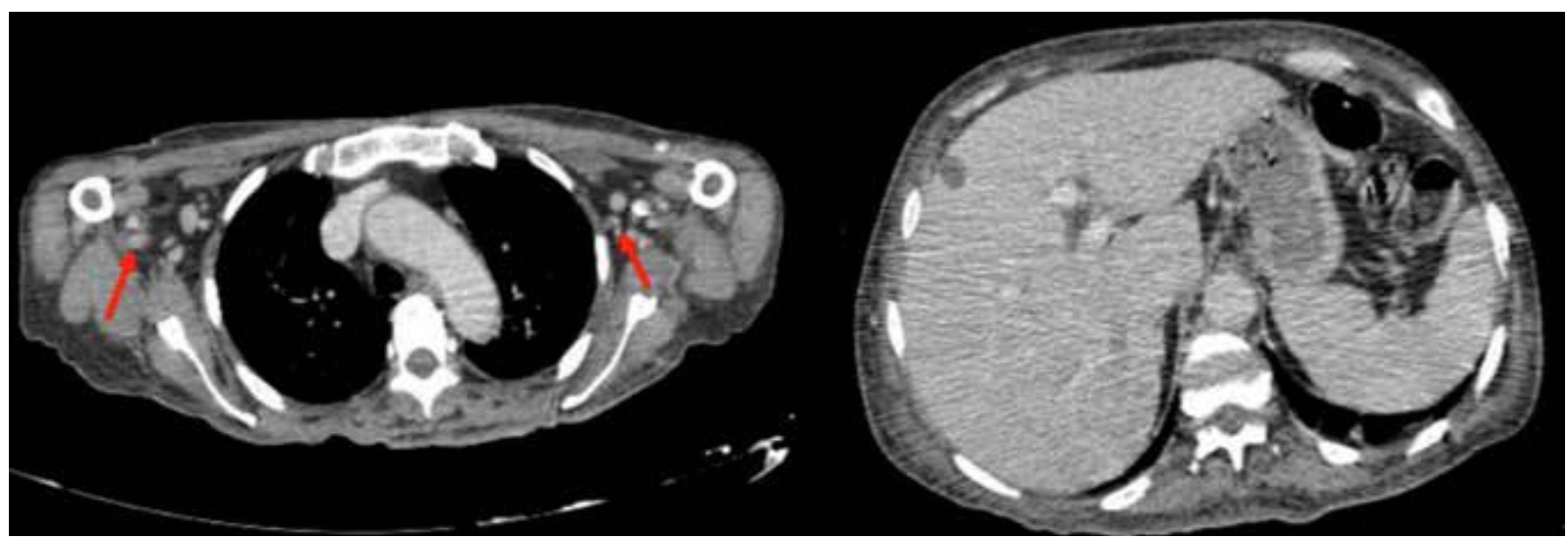

Figura 2.TAC con contraste.A) Ganglios axilares. B) Higado con algunos quiste simples. 

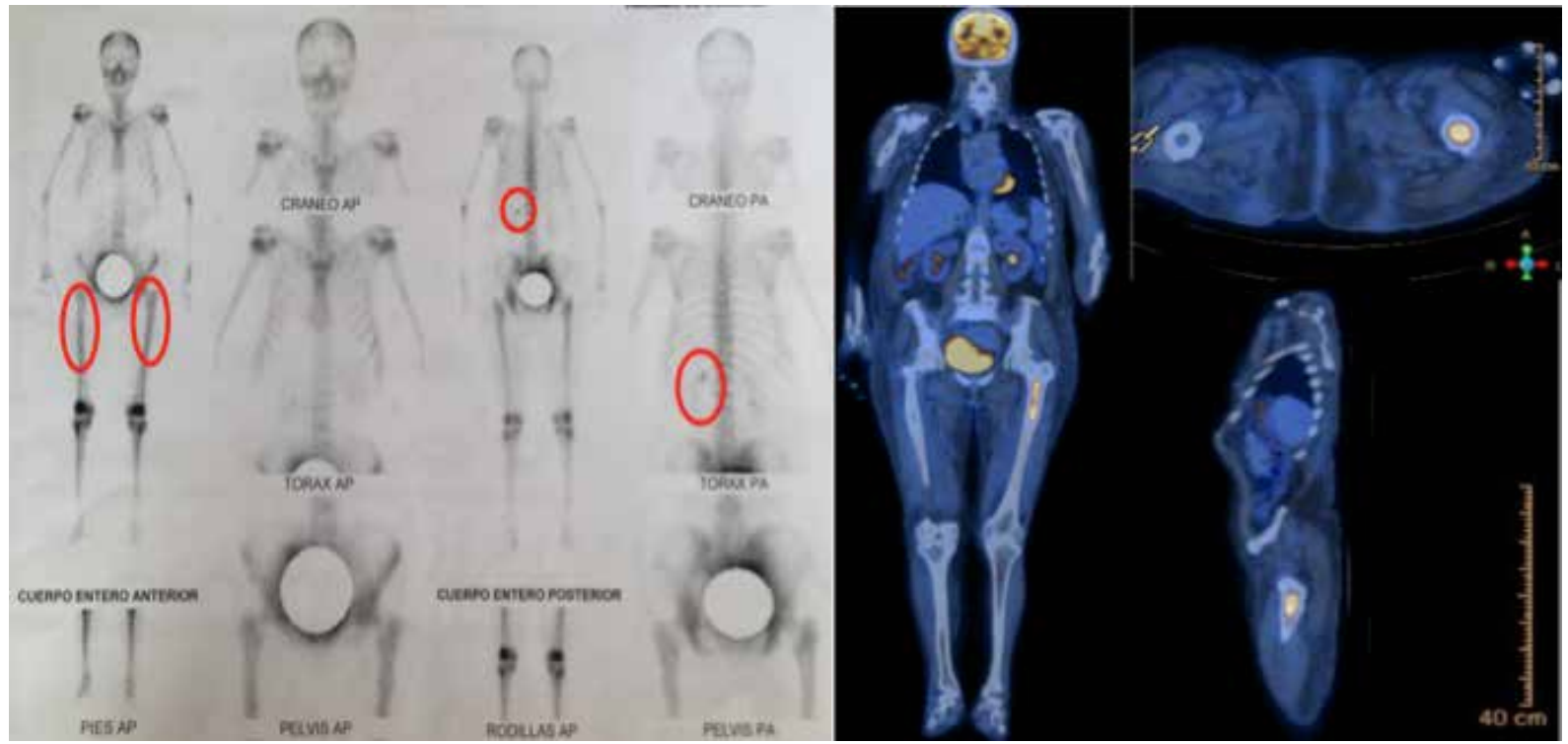

Figura 3.A) Gammagrafía: hipercaptación anormal. B) PET. Scan: hipermetabolismo focalizado en el fémur izquierdo y en el 12 arco costal posterior izquierdo.

puede ser agresiva y se asocia a una mayor morbilidad y mortalidad. Puede virar a linfoma plasmablástico. La causa es multifactorial y en muchos casos son idiopáticos. La ECM asociada a VHH-8 casi siempre está relacionada a inmunosupresión, en especial VIH. En estos pacientes, el VHH-8 es la causa etiológica bien establecida. El VHH-8 infecta las células B y los blastos de los ganglios linfáticos. El virus sintetiza una proteína homologa a la IL6 denominada IL6 viral (vIL6), que desencadena la cascada inflamatoria responsable de la sintomatología. ${ }^{15-17}$

LaECMasociada aPOEMS es un síndrome paraneoplásico debido a un trastorno de las células plasmáticas. La patogénesis exacta aún no se conoce pero algunas citocinas juegan un rol muy importante. Los niveles séricos de VEGF aumentan notablemente y se correlacionan con la actividad de la enfermedad; la IL-1B, TNF y los niveles de IL-6 también aumentan. La neuropatía es la característica dominante del síndrome, comienza como neuropatía sensorial, periférica, ascendente, simétrica y el dolor puede ser una característica dominante. En $95 \%$ de los pacientes hay lesiones osteoscleróticas, y la búsqueda de lesiones en medula ósea debe ser exhaustiva. Es común encontrar edema periférico, derrames pleural y pericárdico y ascitis. Hasta $75 \%$ de los pacientes pueden tener organomegalia. De $11 \%$ a $30 \%$ de los pacientes con POEMS que tienen trastorno de células plasmáticas clonales presentan EC. La anomalía endocrina más común es el hipogonadismo, seguida de anomalías de tiroides, páncreas y suprarrenales. La piel se afecta con hiperpigmentación. Al menos un tercio de los pacientes presentan edema de papila. La hipertensión pulmonar está presente en $25 \%$ de los casos y la enfermedad renal,

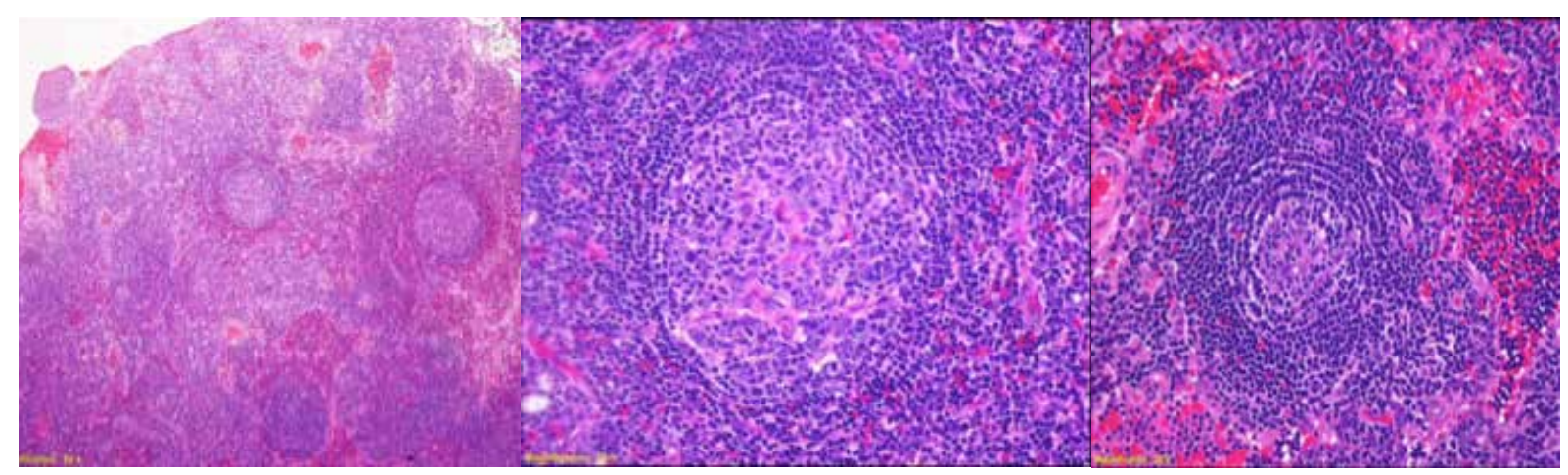

Figura 4. Ganglio axilar: folículos linfoides hipertróficos e hipotróficos, e hiperplasia concéntrica de la zona del manto. 
Tabla I. Criterios diagnósticos del síndrome POEMS.

- Criterios mandatorios

I. Polineuropatía (típicamente desmielinizante)

2. Trastorno proliferativo de células plasmáticas monoclonales (casi siempre lambda)

- Criterios principales (uno requerido)

I. Enfermedad de Castleman

2. Lesiones osteoscleróticas

3. Incremento de los niveles del factor de crecimiento de endotelio vascular (VEGF)

- Criterios menores

I. Organomegalia

2. Sobrecarga de volumen extravascular (edema, efusión pleural, ascitis)

3. Endocrinopatía

4. Cambios en la piel

5. Papiledema

6. Trombocitosis, policitemia.

- Otros síntomas y signos. Pérdida de peso, hiperhidrosis, hipertensión pulmonar, enfermedad pulmonar restrictiva, trombosis, diarrea, valores bajos de vitamina $B / 2$

Fuente: Dispenzieri A.Am J Hematol. 2015;90(10):953.

en menos de $10 \%$. Los pacientes tienen mayor riesgo de trombosis arterial y venosa, infarto de miocardio, accidente cerebrovascular y síndrome de Budd-Chiari. ${ }^{18}$

El diagnóstico de POEMS se confirma con presencia de los dos criterios mandatorios, uno de los tres criterios principales y uno de los seis criterios menores..$^{19}$ Tabla 1.

El tratamiento del POEMS puede estar dirigido a uno de los tres objetivos siguientes: las células clonales específicamente; las citocinas, sobre todo VEGF; la mejora del cuadro neurológico. Se propone un algoritmo de tratamiento basado en la existencia o ausencia de la afectación de la médula ósea: si hay compromiso de médula ósea la terapia debe ser sistémica; si no hay compromiso de medula ósea y tiene de una a tres lesiones óseas debe recibir radioterapia; si hay mayor número de lesiones debe recibir tratamiento sistémico, que puede ser con melfalán más dexametasona, talidomida más dexametasona, lenalidomida más dexametasona o regímenes de bortezomib y la posibilidad del trasplante autólogo de médula ósea con buena respuesta y tasas altas de sobrevivencia. ${ }^{20-22}$

El síndrome TAFRO corresponde a un subtipo de ECMi caracterizado por trombocitopenia $(\mathrm{T})$, anasarca (A), fiebre $(\mathrm{F})$, fibrosis reticulina $(\mathrm{R}) \mathrm{y}$ organomegalia (O). La presencia de trombocitopenia en ausencia de hipergammaglobulinemia sugiere este síndrome en el contexto del hallazgo patológico de EC. ${ }^{23}$
En el presente caso, se encontró electroforesis de proteínas en suero con componente monoclonal de clase $\operatorname{IgG}$ tipo lambda (criterio mandatorio). La electromiografía y la velocidad de conducción con signos de polineuropatía avanzada sensitivo-motora de tipo axonal activa y con escasos signos de reinervación (criterio mandatorio). La biopsia de ganglio axilar fue consistente con EC, variante plasmocelular (criterio principal). La gammagrafía ósea y la PET/CT hallaron compromiso óseo de tipo osteoblástico (criterio principal). Además, organomegalia, endocrinopatía (hipogonadismo e hipotiroidismo subclínico), edema y cambios en la piel como criterios menores.

Con la presencia de dos criterios mandatorios más dos criterios principales y cuatro criterios menores, se hizo el diagnóstico de POEMS. La paciente fue transferida al servicio de Hematología, para el tratamiento especializado, que en este caso fue trasplante de médula ósea, y cursó con buena evolución clínica.

\section{REFERENCIAS BIBLIOGRÁFICAS}

I. Mauermann ML.The peripheral neuropathies of POEMS Syndrome and Castleman Disease. Hematol Oncol Clin NAm. 2018:32:153-163.

2. Kyle RA, Therneau TM, Rajkumar SV, et al. Prevalence of monoclonal gammopathy of undetermined significance. N Engl J Med. 2006;354: I362-9.

3. DispenzieriA. POEMS syndrome: 2017 Update on diagnosis, risk stratification, and management.Am J Hematol. 2017;92:814-829.

4. Arimura K, Hashiguchi T. Crow-Fukase syndrome: clinical features, pathogenesis and treatment in Japan. In:Yamamura T, Kira J,Tabira T, eds. Current topics in neuroimmunology. Bologna, Italy: Medimond, 2007:24I-5.

5. Shi X, Hu S, Luo X, Luo M, You H, Zhu Y, Xi X. CT characteristics in 24 patients with POEMS syndrome.Acta Radiol. 2016:57(I):51-7.

6. Watanabe O, Maruyama I,Arimura K, Kitajima I,Arimura H, Hanatani M, et al. Overproduction of vascular endothelial growth factor/vascular permeability factor is causative in Crow-Fukase (POEMS) syndrome. Muscle Nerve. 1998;21:1390-7.

7. Nobile-Orazio E,Terenghi F, Giannotta C, Gallia F, NozzaA.SerumVEGF levels in POEMS syndrome and in immune-mediated neuropathies. Neurology. 2009;72(II):1024-6.

8. Dao LN, Hanson CA, Dispenzieri A, Morice WG, Kurtin PJ, Hoyer JD. Bone marrow histopathology in POEMS syndrome: a distinctive combination of plasma cell, lymphoid and myeloid findings in 87 patients. Blood. 201 I; I I 7(24):6438-44

9. Dispenzieri A. Castleman disease. Cancer Treat Res 2008;142:293-330.

10. CASE records of the Massachusetts General Hospital Weekly Clinicopathological exercises: case 400I. N Engl J Med. 1954;250(I):26-30.

II. Castleman B, Iverson L, Menendez VP. Localized mediastinal lymphnode hyperplasia resembling thymoma. Cancer. 1956;9(4):822-30.

12. Fajgenbaum DC, Shilling D. Castleman Disease Pathogenesis. Hematol Oncol Clin NAm. 2018;32:II-21.

13. Simpson D. Epidemiology of Castleman disease. Hematol Oncol Clin NAm. 20 I8:32:1-10.

14. Wong RSM. Unicentric Castleman disease. Hematol Oncol Clin N Am. 2018;32:65-73. https://doi.org//0.1016/j.hoc.2017.09.006

15. Kishimoto T.IL-6: from its discovery to clinical applications. Int Immunol. 2010;22:347-52

16. Dossier A, Meignin V, Fieschi C, Boutboul D, et al. Human herpesvirus 8-related Castleman disease in the absence of HIV infection. Clin Infect Dis. 2013;56(6):833-42

17. Suda T, Katano H, Delsol G, Kakiuchi C, et al. HHV-8 infection status of AIDS-unrelated and AIDS-associated multicentric Castleman's disease. Pathol Int. 2001;51:67I-9.

18. DispenzieriA, Kourelis T,Buadi F.POEMS Syndrome:Diagnosis and InvestigativeWork-up. Hematol Oncol Clin NAm. 2018;32:1 19-139. https://doi.org/I0.1016/j.hoc.2017.09.010

19. Am J Hematol. 2015;90(10):953

20. Dispenzieri A. POEMS syndrome. Blood Rev. 2007;21:285-99.

21. Dispenzieri A. POEMS syndrome: 2017 update on diagnosis, risk stratification, and management.Am J Hematol. 2017;92:8।4-29.

22. Jaccard A. POEMS syndrome: therapeutic options. Hematol Oncol Clin N Am. 2018;32:141-151.https://doi.org//0.1016/j.hoc.2017.09.01।

23. Takai KI,et al. [Thrombocytopenia with mild bone marrow fibrosis accompanied by fever, pleural effusion, ascites and hepatosplenomegaly.] Rinsho Ketsueki. 2010;51:320-5.

CORRESPONDENCIA: Eloy Peña

eloy.pena.m@upch.pe

FECHA DE RECEPCIÓN: I5 de julio de 2019.

FECHA DE ACEPTACIÓN: 30 de julio de 2019 